\title{
Involvement of neutrophils in exercise-induced muscle damage and its modulation
}

\author{
Katsuhiko Suzuki* \\ Faculty of Sport Sciences, Waseda University, Japan
}

\begin{abstract}
Neutrophils not only play a critical role in host defense by migrating to the site of infection and producing reactive oxygen species (ROS), but also mediate pathological processes involved in tissue destruction and inflammatory diseases. Therefore, it is important to assess and modulate neutrophil activities. In the present article, I would like to share some of our research on neutrophils in relation with exercise and muscle damage. I will begin with my early studies on neutrophil functional analyses and a newly developed measurement system. Then, some key data about effects of exercise, antioxidant modulation, mechanisms of exerciseinduced muscle damage, and possible preventive countermeasures targeting pathogenesis are described herein.
\end{abstract}

\section{Introduction}

Polymorphonuclear (PMN) or neutrophilic leukocytes (neutrophils), a kind of white blood cell, make up 50 to $60 \%$ of blood leukocytes (Figure 1) and they have a short life span of 6 to $20 \mathrm{~h}$. Upon infection, they rapidly move to the site of inflammation and ingest microbes and cell debris in phagosomes. Especially, reactive oxygen species (ROS) are produced, which are involved in killing microbes and tissue damage. Neutrophils work in the initial stages of inflammation and immune response.

In 1990, I started doing functional analyses of neutrophils using the histochemical nitroblue tetrazolium (NBT) test [1,2], but it was difficult to use to search for neutrophils on blood smears. Also, it was not a quantitative measurement, although we could see phagocytosis of microbes and black deposition, which reflects ROS production that causes self-destruction and surrounding erythrocyte deformation and damage (Figure 2).

In 1993, I started medical school, and had a chance to develop a new simultaneous and quantitative chemiluminescence measurement system for comparing many experimental conditions and samples of neutrophils $[3,4]$. This took high-sensitivity measurements by photon counting with a cooled CCD camera. It allowed for simultaneous measurements of multiple samples in a 96-well microplate, under regular intermittent shaking and at $37^{\circ} \mathrm{C}$.

At first, we applied these advantages to the assessment of micronutrient actions. Dr. Hasegawa, the first author of our paper that described development of the measurement system [4], was engaged in a study of zinc [5]. Then, Dr. Liu, who had written many papers on antioxidant and pharmacological agents [6-8], became a professor at China medical university soon after returning to China. I was a medical student at the time and was engaged in exercise studies, because neutrophil dynamics can be used as sensitive markers of exercise stress [9-11]. We published many papers together on bioactive agents in vitro, and stress reactions in vivo and ex vivo [12-14].

For example, we investigated the effects of 90-min of bicycle exercise at the same anaerobic threshold (AT) level for 3 consecutive days on circulating leukocyte and neutrophil counts and on neutrophil degranulation [9]. On Day 1, total leukocytes increased dramatically due to neutrophils and there was degranulation of PMN elastase in the circulation (Figure 3). This showed neutrophil mobilization and activation following acute endurance exercise. However, these responses were decreased on Days 2 and 3. When we measured the capacity of neutrophils to produce ROS by luminol-dependent chemiluminescence with ex vivo stimulation using opsonized zymosan and phorbol myristate acetate (PMA), we found remarkable increases on Day 1 only (Figure 4). Similarly, there was a marked increase in the muscle damage marker, myoglobin, after exercise on Day 1 only (Figure 5). The correlations seen among the variables suggested the involvement of neutrophils in muscle damage and the involvement of interleukin (IL)-6 in neutrophil mobilization $[9,10]$.

Besides this study, we had investigated the effects of various exercise conditions on neutrophils. We found that augmented ROS production and degranulation depended on exercise intensity and duration [1420]. It was also suggested that exercise-induced neutrophil mobilization and activation might be associated with muscle damage, but these responses were decreased by daily repetition of exercise and training $[9,17,19]$, suggesting some anti-inflammatory mechanisms of exercise/ training. Indeed, it was demonstrated using this measurement system $[3,4]$ that post-exercise serum samples have much stronger potentials to suppress neutrophil and monocyte activation [11].

However, there were several technical issues at that time. First, to measure neutrophil functions, we needed to separate neutrophils from whole blood (Figure 6). Also, great care was needed to do this. It took at least $1 \mathrm{~h}$ to adjust a fixed cell concentration of neutrophils for

${ }^{\star}$ Correspondence to: Katsuhiko Suzuki, Faculty of Sport Sciences, Waseda University, Japan, E-mail: katsu.suzu@waseda.jp

Key words: polymorphonuclear (PMN) leukocyte, myeloperoxidase (MPO) granulocyte, macrophage, oxidative stress, inflammation, antioxidant intervention

Received: November 12, 2018; Accepted: November 22, 2018; Published: November 27, 2018 


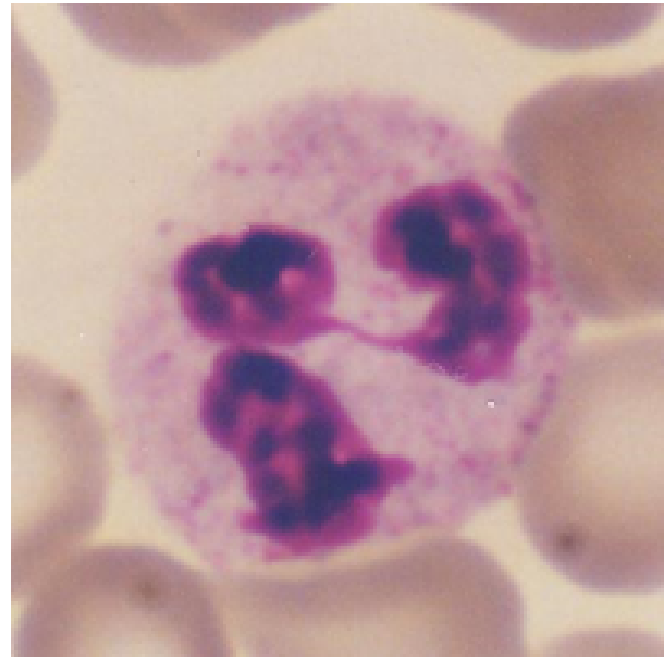

Figure 1. Neutrophils shown on stained blood smears are characterized as polymorphonuclear (PMN) leukocytes containing neutrophilic granules (granulocytes) which include PMN elastase and myeloperoxidase (MPO)
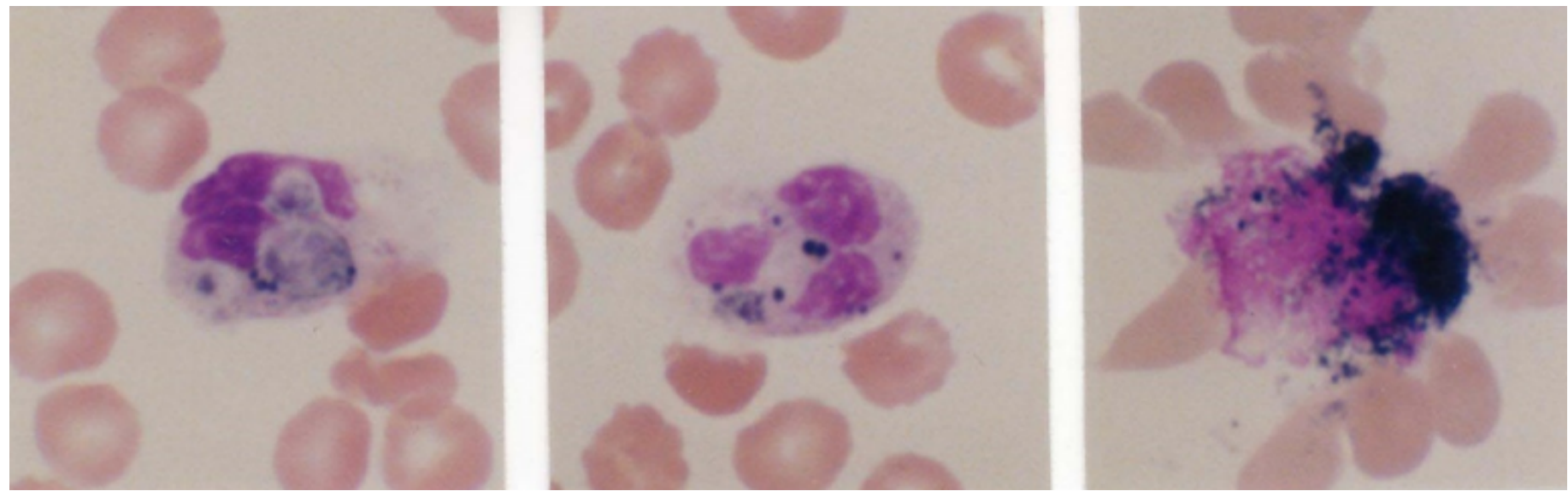

Figure 2. Neutrophils ingest zymosan (left), Staphylococcus. aureus (central), and were damaged by overproduction of reactive oxygen species (ROS) upon stimulation with phorbol myristate acetate (PMA) together with surrounding erythrocyte deformation and damage (right). Black deposition reflects ROS production [16]

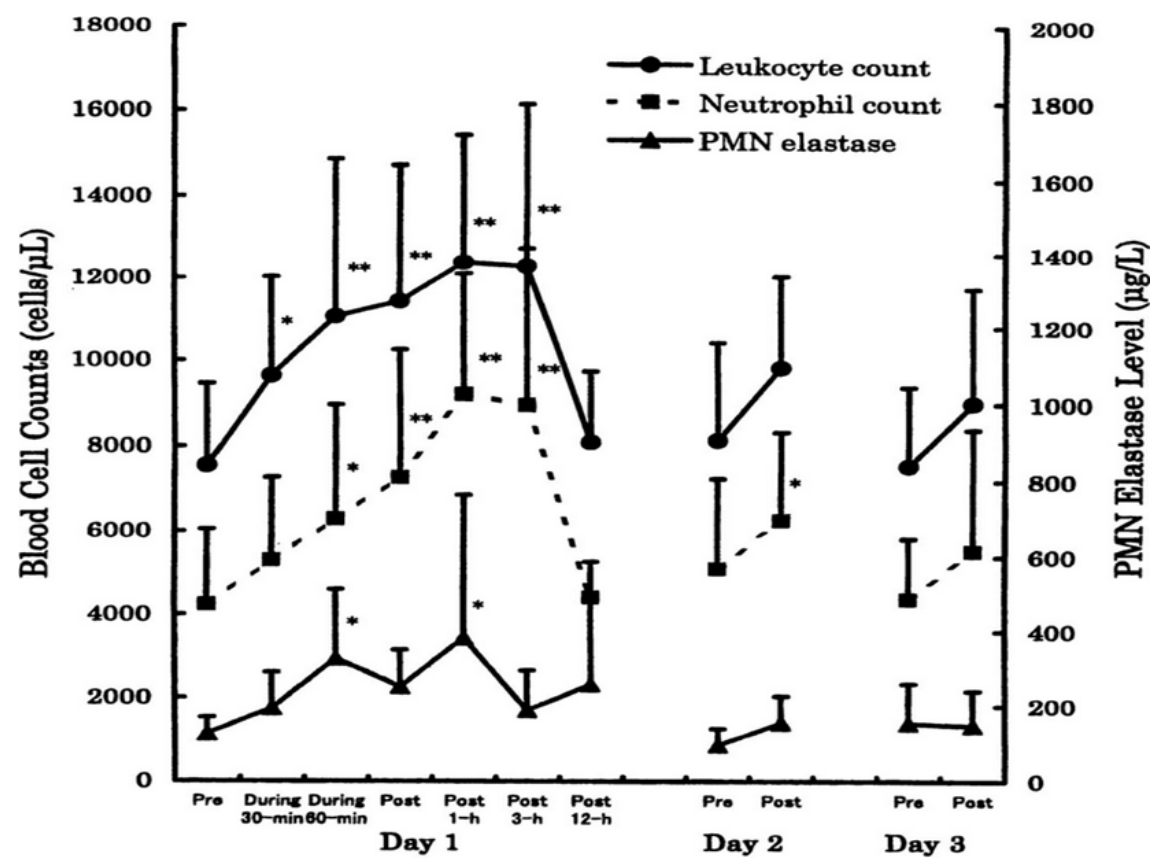

Figure 3. Effects of same 90-min anaerobic threshold (AT) level bicycling for 3 days on circulating leukocyte and neutrophil counts and degranulation of neutrophils in men [9] 


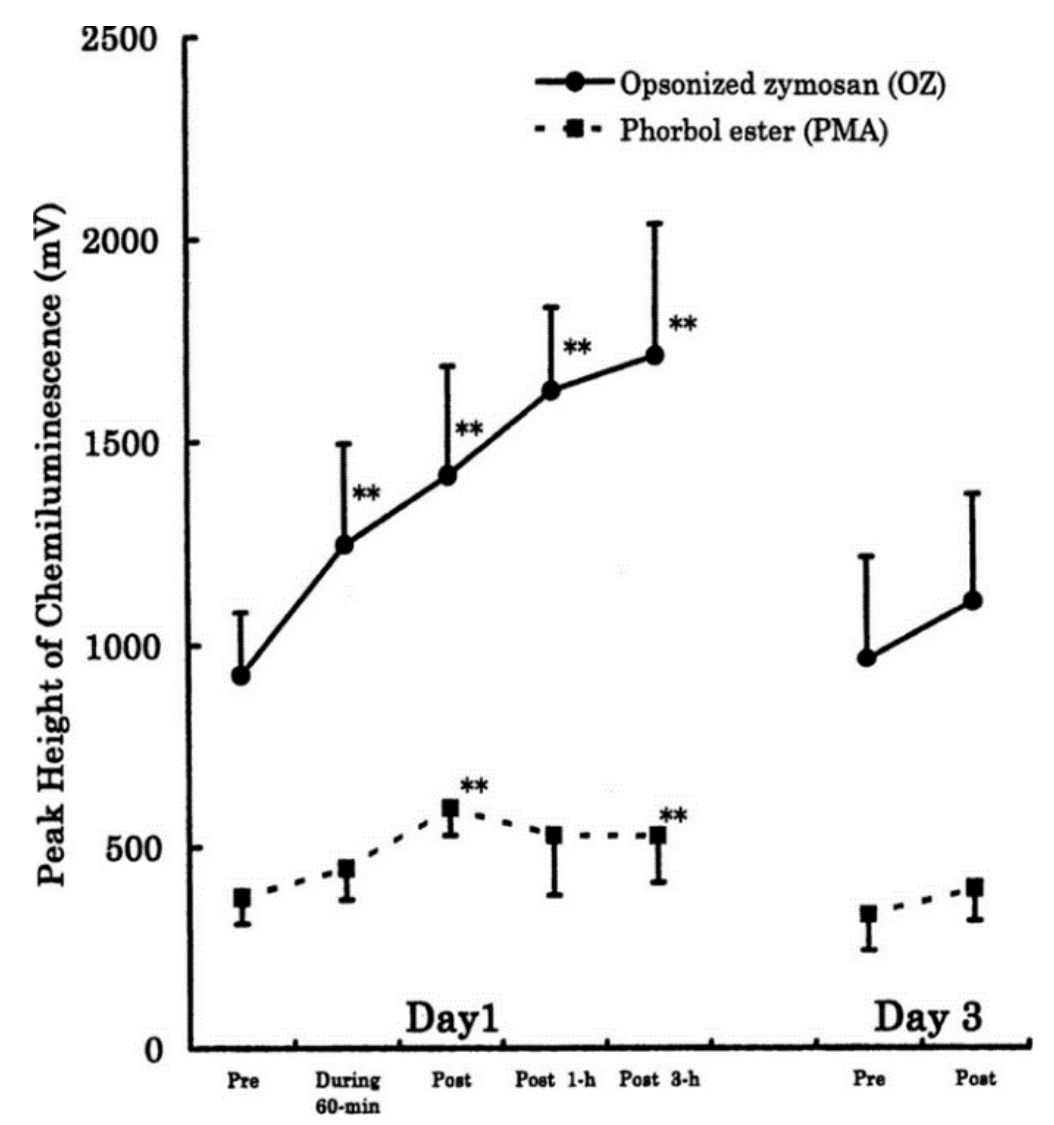

Figure 4. Capacity of circulating neutrophils to produce ROS following same 90-min AT level bicycling for 3 days in men. Neutrophil activity was measured by ex vivo stimulation using luminol-dependent chemiluminescence [9] which monitors MPO-mediated formation of highly toxic oxidants such as OCl- and ONOO- $[4,12,16]$

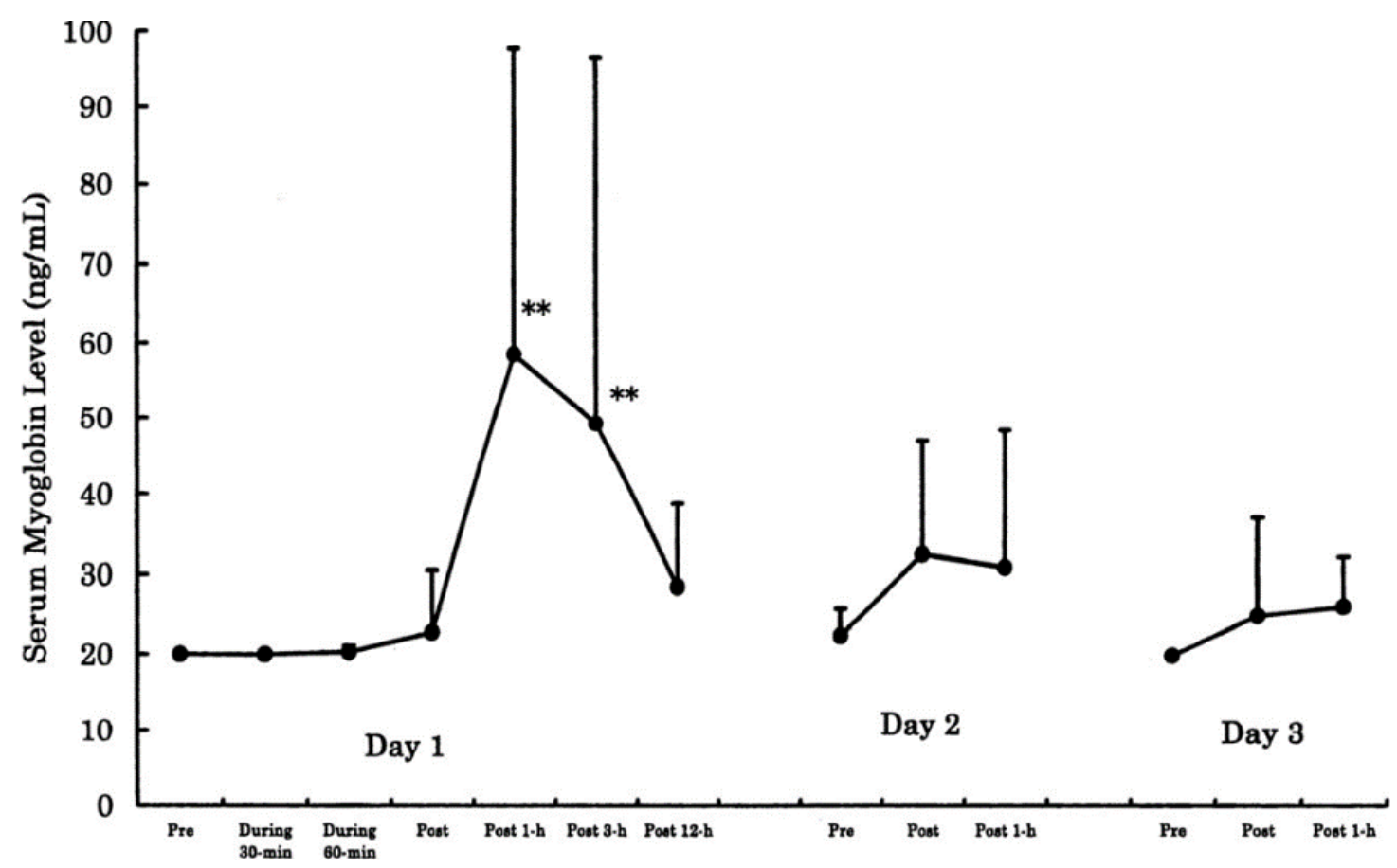

Figure 5. Changes of muscle damage marker, serum myoglobin level, following same 90-min AT level bicycling for 3 days in men [9] 


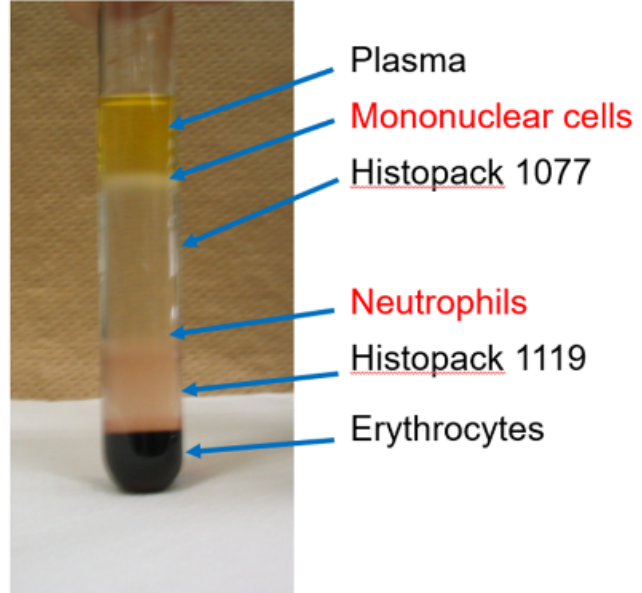

Figure 6. Isolation of neutrophils with the density gradient centrifugation method

the functional analyses, and some researchers criticized the method, saying that the recovery rate was low and the neutrophil functions were altered from the in vivo environments.

\section{Development of new measurement system of neutrophil functions}

In 2003, when I moved to Waseda University, I started trying to solve these technical issues $[11,20]$. We started with developing a new measurement system of neutrophil activity. We used hydrogel material to mimic in vivo microenvironments (Figure 7).

To improve neutrophil migration, we had the chance to try a new polymer. Researchers at Waseda University's Faculty of Science and Engineering had developed thermoreversible gelation polymer (TGP) $[21,22]$, which shows the interesting property, where it is a solution at low temperature and a gel at higher temperature, which is something reversed with Chinese isinglass or Japanese agar. The molecular design of the material is a repeating chain of thermo-responsive polymers and hydrophilic polymers (Figure 8). At less than $20^{\circ} \mathrm{C}$, TGP is a fluid, a solution, but above $20^{\circ} \mathrm{C}$, it is a gel because of the thermoresponsive polymer. To attract neutrophils, we tried to mix TGP with chemoattractants such as IL-8 and extracellular matrixes.

For neutrophil functional measurements, we used heparinized whole blood samples. TGP was used for neutrophil migration $[21,22]$, and luminol was used for measuring neutrophil-specific ROS production $[4,16]$. First, the cooled solution was put into the tube and warmed to $37^{\circ} \mathrm{C}$. This resulted in a gel that firmly attached to the bottom of the tube. Then, heparinized blood and luminol were mixed, and the chemiluminescence signal was monitored without the isolation of neutrophils by density gradient centrifugation (Figure 6). This new method enabled us to start measurements immediately after blood sampling. When the mixture of blood and luminol is added on the gel, neutrophils start to migrate to the gel and emit chemiluminescence in the transparent hydrogel, which can be detected by a photomultiplier at the bottom of the tube (Figure 7). If there is any chemiluminescence in the blood, it can be quenched by massive red blood cells (Figure 7). After measuring chemiluminescence for $1 \mathrm{~h}$, whole blood can be removed with warmed phosphate buffered saline (PBS), leaving the migrated neutrophils. By cooling down the tube, the migrated cells are suspended in solution and the cell count can be quantified with a hematocytometer or automatic cell counter.

\section{Effects of endurance exercise on neutrophil functions}

We then used this measurement system to investigate the effects of intense endurance exercise [23]. Because we had already reported that exhaustive endurance exercise causes neutrophil mobilization and priming that might be associated with muscle damage [9,15-19], we examined neutrophil activity ex vivo using our newly developed neutrophil function measurement system.

Fourteen male triathletes completed a duathlon race involving $5 \mathrm{~km}$ running, $40 \mathrm{~km}$ cycling, and $5 \mathrm{~km}$ running. Blood and urine samples were collected before and after the race. The mean time of the race was around $2 \mathrm{~h}$. Part of the blood sample was used for plasma and serum separation. Whole blood was mixed with luminol, and neutrophil migration into the gel and ROS production were determined. In other words, by using hydrogel to mimic damaged muscle tissue, we could check the ability of neutrophils from freshly drawn blood to migrate and produce ROS ex vivo (Figure 7). We also measured other variables using whole blood, plasma, serum, and urine $[23,24]$.

After the race, we observed increases in migratory neutrophils and ROS production in the hydrogel. There were also dramatic increases in plasma levels of IL-6, myeloperoxidase (MPO), and myoglobin. Although there were no marked changes in IL-17, IL-23 and sRANKL,

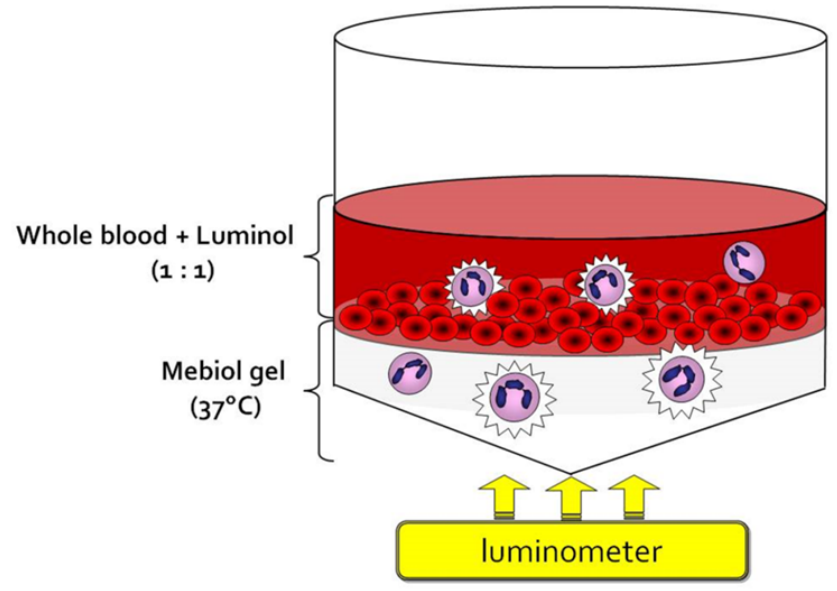

Figure 7. Novel measurement system of neutrophil activity ex vivo [21]
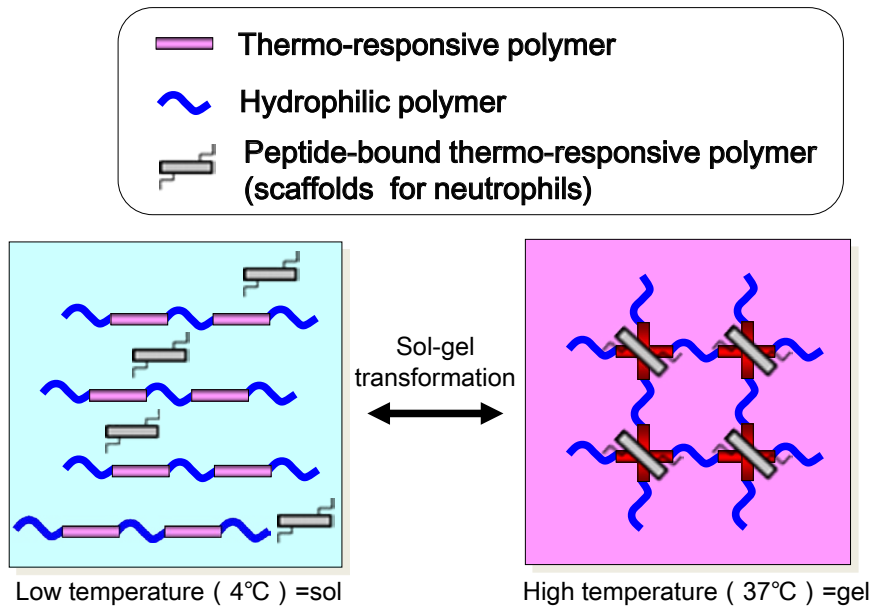

Figure 8. Sol-gel transformation of thermoreversible gelation polymer (TGP) [22] 
some other cytokines were increased, especially in urine. These cytokines, muscle damage markers, and MPO were closely correlated (Figure 9). Therefore, in our research paper, we suggested that the systemic inflammatory responses I described earlier [9] were, at least in part, the underlying mechanisms of exercise-induced muscle damage [23,24].

\section{Effects of local eccentric exercise on neutrophil functions}

Delayed-onset muscle soreness (DOMS) occurs several days after unaccustomed or eccentric exercise, but the underlying mechanisms were not clear. Therefore, we examined the associations between muscle soreness, muscle damage markers, and inflammatory mediators [25]. The eccentric exercise protocol involved calf raise exercises. We collected blood and urine samples before and after calf raise exercises, and repeated this process over 4 days. We measured many hematological and biochemical variables, muscle damage markers, and inflammatory mediators including neutrophil functions as described before [25].

DOMS occurred on day 2 and later after exercise. All of the muscle damage markers were increased on day 4 , except for myoglobin which was increased earlier, on day 3 , with better sensitivity. Total leukocyte count was increased $4 \mathrm{~h}$ after exercise, and this increase was due to neutrophils. There were also increases in neutrophil migration into the gel and ROS production. However, other biochemical variables and inflammatory mediators showed no change at all, unlike the findings for endurance exercise $[23,24]$. Nevertheless, we found correlations between the muscle damage marker myoglobin and muscle soreness and between neutrophil migration and myoglobin [25].

This study demonstrated that circulating myoglobin concentration was the most sensitive among the muscle damage markers, and it was closely correlated with DOMS. Because circulating neutrophil responses to exercise increased $4 \mathrm{~h}$ after exercise, this suggests that neutrophils mobilized into the circulation can migrate to the muscle tissue affected by eccentric exercise, and result in muscle damage and inflammation.

\section{In vitro actions of antioxidants and anti-inflammatory agents}

Although we had confirmed that our measurement system was more sensitive than other inflammatory markers, we decided to also check the in vitro actions of antioxidants and anti-inflammatory agents. The most representative antioxidant, vitamin C, did not reduce neutrophil migration but did reduce ROS in a concentration-dependent manner. On the other hand, the typical anti-inflammatory agent hydrocortisone

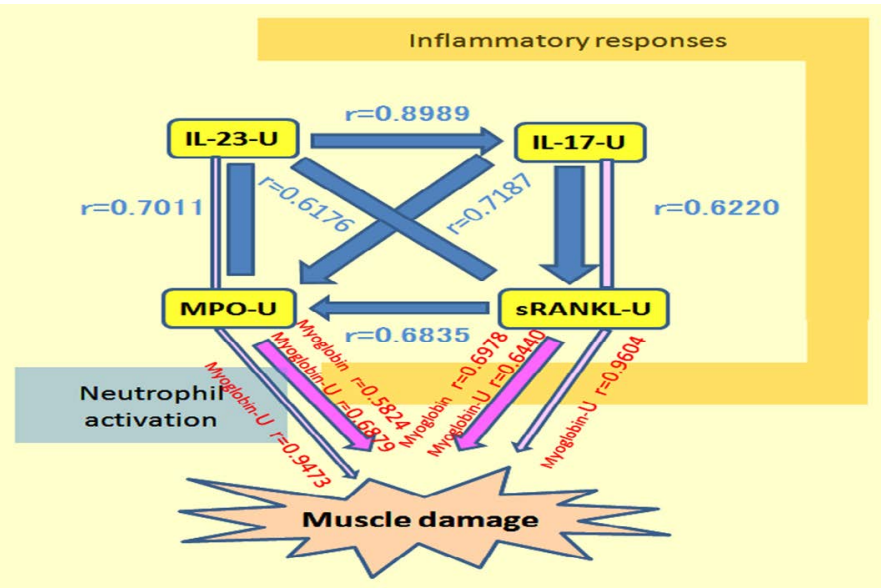

Figure 9. Correlations of inflammatory responses and neutrophil activation [24]
Table 1. In vitro effects of anti-oxidative and anti-inflammatory substances on neutrophil activity

\begin{tabular}{|c|c|c|}
\hline & Migration & ROS Production \\
\hline \multicolumn{3}{|l|}{ Antioxidants } \\
\hline Vitamin C & $\rightarrow$ & $\downarrow$ \\
\hline Polyphenols & $\rightarrow$ & $\downarrow$ \\
\hline Catechins & $\rightarrow$ & $\downarrow$ \\
\hline \multicolumn{3}{|c|}{ Anti-inflammatory drugs } \\
\hline Hydrocortisone & $\downarrow$ & $\downarrow$ \\
\hline Epinephrine & $\rightarrow$ & $\rightarrow$ \\
\hline
\end{tabular}

reduced both neutrophil migration and ROS production. We checked many other substances as well $[26,27]$. Only antioxidants reduced ROS production in a concentration-dependent manner, but some antiinflammatory agents reduced neutrophil migration (Table 1).

Here, I will mention the interesting findings for curcumin, which is a kind of polyphenol found in turmeric. Recently, the effectiveness of curcumin was demonstrated in a wide variety of diseases related with acute and chronic inflammation [28]. As for its mechanisms of action, it acts on many inflammatory substances, but it was not known to act on neutrophil functions at that time. We showed that curcumin attenuated neutrophil migration in subjects with higher responsiveness, and it attenuated ROS production in all subjects (Figure 10). These findings suggest that curcumin is an antioxidant with some anti-inflammatory action [22].

\section{Effects of curcumin intake on exercise-induced oxidative stress}

We then conducted a human study on the effects of curcumin on exercise-induced oxidative stress [29]. Ten male students did treadmill running for $60 \mathrm{~min}$ at $65 \%$ maximal oxygen uptake in a double-blind cross-over design of placebo intake, single intake just before exercise, and double intake before and after exercise. In the placebo trial, exercise caused oxidative stress, but this was prevented by curcumin intake before exercise.

Also, we conducted animal studies because we cannot obtain information on intramuscular events in humans for ethical reasons. Dr. Kawanishi investigated the effects of curcumin ingestion on muscle damage induced by downhill running in mice [30]. After 2.5 $\mathrm{h}$ of downhill running, curcumin or PBS was orally administered, and muscle was sampled $24 \mathrm{~h}$ later. Downhill running caused production of ROS in damaged muscle, but curcumin intake prevented it. As for chemokines, MCP-1 gene expression showed similar patterns. Macrophage infiltration was also attenuated by curcumin administration. To sum up, curcumin inhibited MCP-1 production, and it reduced macrophage infiltration and ROS production. Although the involvement of neutrophils was not examined in the study, it was confirmed that leukocytes could be one of the targets for the prevention of oxidative stress and inflammation in the exercise-induced muscle damage [31-33].

\section{Role of neutrophils in the exercise-induced muscle damage}

Dr. Kawanishi also led our investigation into the involvement of neutrophils and macrophages in exhaustive exercise-induced muscle damage and inflammation [34,35]. I will outline the definitive evidence we obtained using a mouse model. We know that prolonged exercise causes muscle damage, and muscle membrane permeability can be seen on immunohistochemistry using dystrophin and IgG staining. In the mouse model, in the sedentary condition, neutrophil and macrophage counts were low in skeletal muscle, but they were 

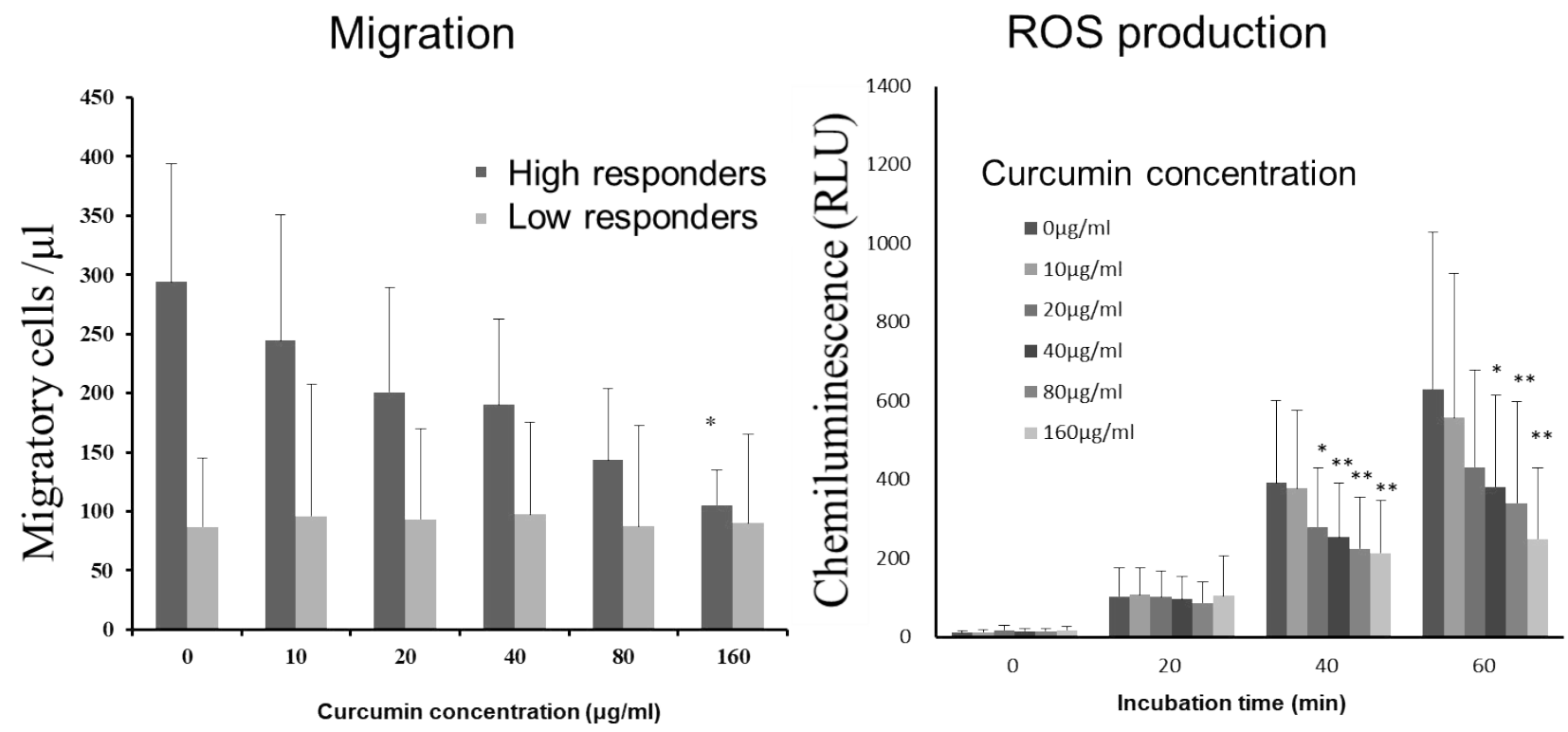

Figure 10. Effect of curcumin on neutrophil functions in vitro [22]

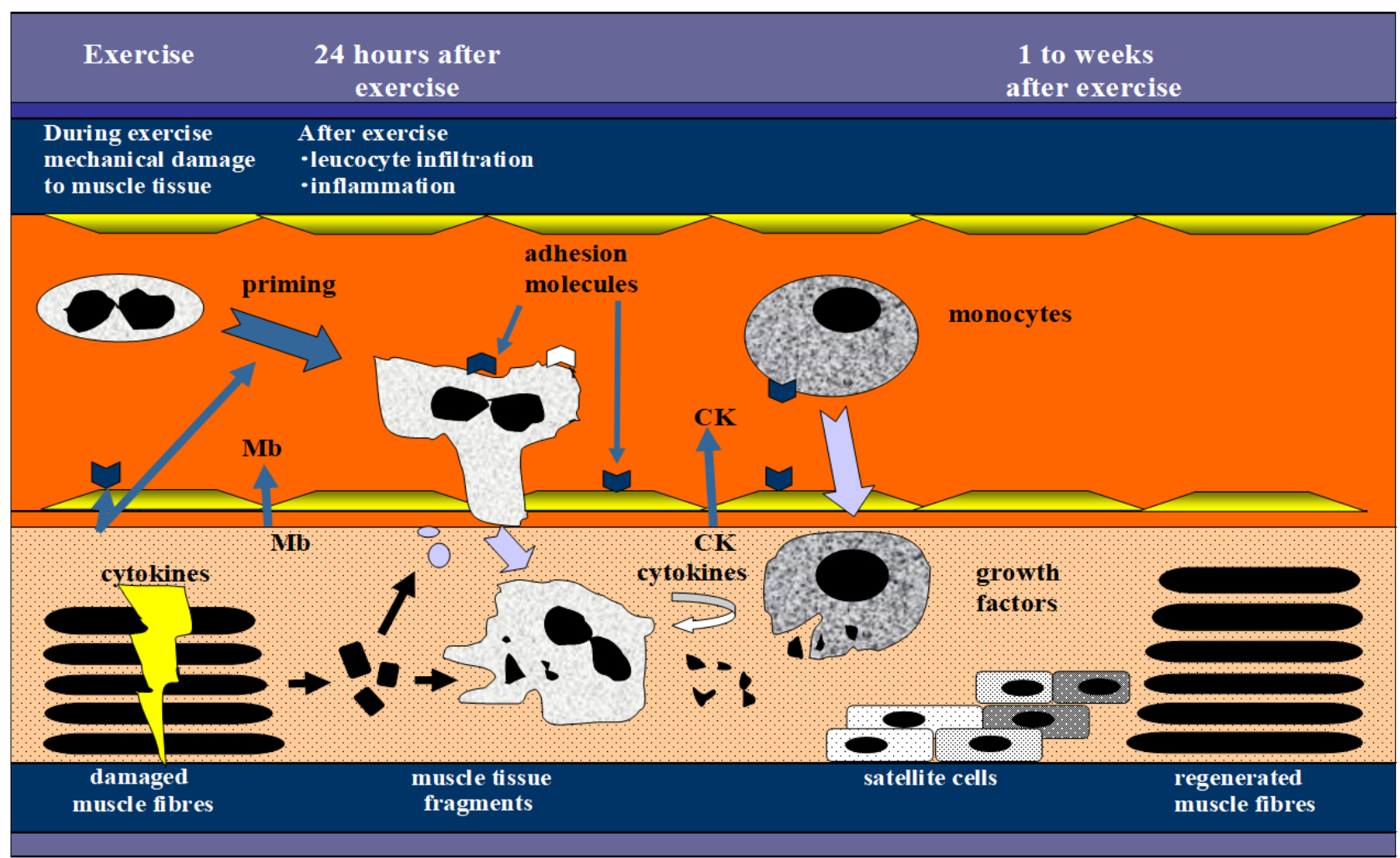

Figure 11. Accumulation of neutrophils and macrophages contribute to the mechanisms of muscle injury by regulating inflammation [36]

increased after treadmill running until exhaustion. However, we could reduce myofiber injury and proinflammatory cytokines by depleting neutrophils before exercise -with an intraperitoneal injection of antineutrophil antibody [34]. A similar finding was seen by depleting macrophages with an intraperitoneal clodronate liposome injection [35]. These findings indicate that exhaustive exercise caused neutrophil accumulation and chemokine release in skeletal muscle, which induced macrophage accumulation and proinflammatory cytokine production, and resulted in myofiber injury. These studies provided experimental evidence of the involvement of neutrophils in exercise-induced muscle damage, as modeled previously by us [36] (Figure 11).

\section{Related and supplementary findings}

Aside from functional food components such as curcumin, reducing exercise-induced dehydration by sports drink ingestion also inhibited neutrophil activation and cytokine release in vivo [37, 38]. Although high-dose carbohydrate ingestion after resistance exercise can induce muscle inflammation [39], the ingestion after endurance exercise 

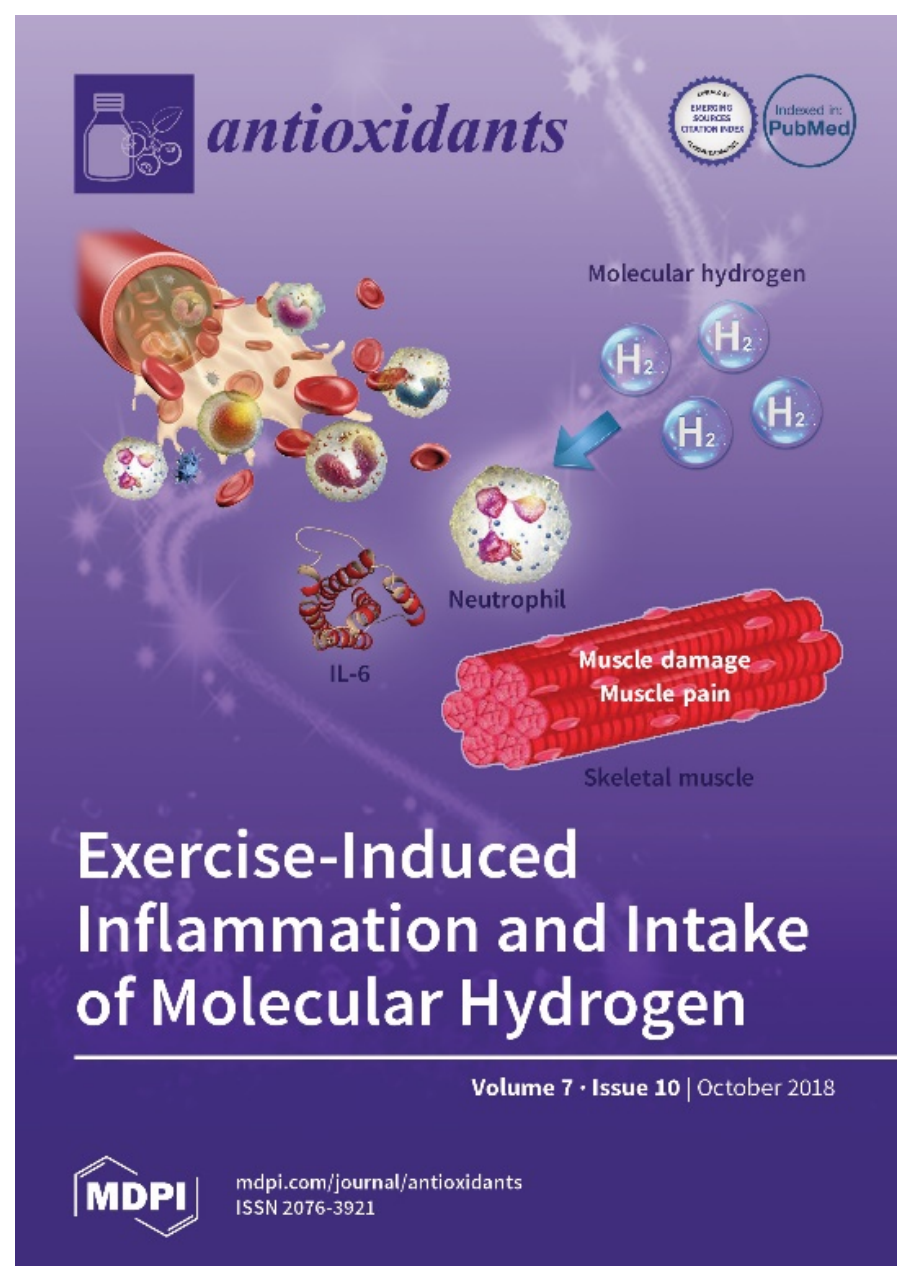

Figure 12. Cover story: IL-6, as a multifunctional cytokine, may be involved in the mobilization of neutrophils into the peripheral blood and subsequent muscle damage, inflammation and DOMS following systemic eccentric exercise. Meanwhile, the intake of molecular hydrogen, known to possess antioxidant and anti-inflammatory effects in the disease model, seems to be ineffective against the inflammatory process centered on neutrophils, following systemic eccentric exercise. Image source: https://www.mdpi. com/2076-3921/7/10/127

does not enhance exercise-induced increase in circulating neutrophil and cytokine levels and markers of neutrophil activation and muscle damage [40]. Cold water immersion after resistance exercise does not reduce exercise-induced inflammation [41]. Downhill running, a mode of dynamic eccentric exercise, causes muscle damage, DOMS, neutrophil mobilization and activation, but molecular hydrogen was not effective on neutrophil activity [42], which was covered by the Antioxidants (Figure 12).

Further studies are needed to investigate which interventional strategies are effective to reduce inflammation, because severe systemic inflammation can cause multiple organ failure and heat stroke $[43,44]$. Therefore, these potential countermeasures not only help to reduce pathophysiological processes, but also lead to new research findings for the prevention of oxidative stress, inflammation, organ damage and dysfunction [44-50].

\section{Concluding remarks}

Strenuous exercise induces leukocytosis mainly due to neutrophilia in the systemic circulation, whereas neutrophil activity is associated with muscle damage and other internal organ dysfunctions.
As for the underlying mechanisms, the research findings of exertional effects on systemic inflammation centered on neutrophils are described above, which are together with cytokines [42,51-53] in line with the pathogenesis of not only muscle but also multiple organ failure in systemic inflammation, heat stroke and sepsis.

Future studies are required to examine the validity of such prevention and treatment approaches, based on their mechanisms of action, focusing on the benefits of measuring neutrophil functional activity and dynamics through translational research.

\section{Acknowledgements}

I greatly appreciate the contribution of graduates from the Preventive Medicine and Applied Immunology laboratory (http:// www.f.waseda.jp/katsu.suzu/english.html) and those involved in the projects. Also, I would like to thank Dr. Llion Roberts, School of Allied Health Sciences \& Menzies Health Institute Queensland, Griffith University, and Pr. Caryn Jones, ThinkSCIENCE, Inc., for English editing.

\section{References}

1. Suzuki K, Machida K, Kariya M (1992) Conditions for low-intensity voluntary wheel running in rats and its chronic effects on health indexes. Jpn J Hyg 47: 939-951.

2. Suzuki K, Machida K, Sekine Y, Murayama R (1993) Analysis and assessment of superoxide productivity of phagocytes using histochemical NBT reduction technique and its application. J Phys Fit Nutr Immunol 3: 31-39.

3. Kikuchi T, Suzuki K, Abe T, Satoh H, Endoh T, et al. (1997) Measurement of chemiluminescence from neutrophils in a 96-well microplate using Lumi Box U-800 II. J Biolumin Chemilumin 12: 149-153. [Crossref]

4. Hasegawa H, Suzuki K, Nakaji S, Sugawara K (1997) Analysis and assessment of the capacity of neutrophils to produce reactive oxygen species in a 96-well microplate format using lucigenin- and luminol-dependent chemiluminescence. $J$ Immunol Methods 210: 1-10.

5. Hasegawa H, Suzuki K, Nakaji S, Sugawara K (2000) Effects of zinc on the reactive oxygen species generating capacity of human neutrophils and on the serum opsonic activity in vitro. Luminescence 15: 321-327. [Crossref]

6. Liu Q, Suzuki K, Kudo S, Yamada M, Kowatari K, et al. (2000) Effect of decaglycerol monooleate on phagocytosis and respiratory burst activity of human neutrophils: an in vitro study. Food Chem Toxicol 38: 432-428.

7. Liu Q, Suzuki K, Nakaji S, Sugawara K (2000) Antioxidant activities of natural 9-cis and synthetic all-trans $\beta$-carotene assessed by human neutrophil chemiluminescence. Nutr Res 20: 5-14. [Crossref]

8. Liu Q, Shimoyama T, Suzuki K, Umeda T, Nakaji S, et al. (2001) Effect of sodium butyrate on reactive oxygen species generation by human neutrophils. Scand $J$ Gastroenterol 36: 744-750.

9. Suzuki K, Totsuka M, Nakaji S, Yamada M, Kudoh S, et al. (1999) Endurance exercise causes interaction among stress hormones, cytokines, neutrophil dynamics, and muscle damage. J Appl Physiol 87: 1360-1367. [Crossref]

10. Yamada M, Suzuki K, Kudo S, Totsuka M, Nakaji S, et al. (2002) Raised plasma G-CSF and IL-6 after exercise may play a role in neutrophil mobilization into the circulation. J Appl Physiol 92: 1789-1794.

11. Suzuki K, Nakaji S, Yamada M, Liu Q, Kurakake S, et al. (2003) Impact of a competitive marathon race on systemic cytokine and neutrophil responses. Med Sci Sports Exerc 35: 348-355. [Crossref]

12. Kudoh S, Suzuki K, Yamada M, Liu Q, Nakaji S, et al. (1999) Contribution of nitric oxide synthase to human neutrophil chemiluminescence. Luminescence 14: 335-339. [Crossref]

13. Kowatari K, Suzuki K, Kudo S, Yamada M, Liu Q, et al. (1999) Applicability of chemiluminescence to assess the degree of operative stress in patients undergoing spinal surgery. Luminescence 14: 331-334. [Crossref]

14. Yamada M, Suzuki K, Kudo S, Totsuka M, Simoyama T, et al. (2000) Effect of exhaustive exercise on human neutrophils in athletes. Luminescence 15: 15-20. [Crossref]

15. Suzuki K, Totsuka M, Naganuma S, Shiraishi M, Mochizuki M, et al. (1994) Effects of endurance exercise on blood neutrophils in sedentary men. J Phys Fit Nutr Immunol 4: 18-24. 
16. Suzuki K, Sato H, Kikuchi T, Abe T, Nakaji S, et al. (1996) Capacity of circulating neutrophils to produce reactive oxygen species after exhaustive exercise. $J$ Appl Physiol 81: 1213-1222. [Crossref]

17. Suzuki K, Naganuma S, Totsuka M, Suzuki KJ, Mochizuki M, et al. (1996) Effects of exhaustive endurance exercise and its one-week daily repetition on neutrophil count and functional status in untrained men. Int J Sports Med 17: 205-212. [Crossref]

18. Sato H, Abe T, Kikuchi T, Endo T, Hasegawa H, et al. (1996) Changes in the production of reactive oxygen species from neutrophils following a $100-\mathrm{km}$ marathon. Nihon Eiseigaku Zasshi 51: 612-616.

19. Mochizuki M, Suzuki K, Nakaji S, Sugawara K, Totsuka M, et al. (1999) Effects of maximal exercise on nonspecific immunity in athletes under trained and detrained conditions. Jpn J Phys Fitness and Sports Med 48: 147-159. [Crossref]

20. Peake J, Wilson G, Hordern M, Suzuki K, Yamaya K, et al. (2004) Changes in neutrophil surface receptor expression, degranulation, and respiratory burst activity after moderate- and high-intensity exercise. J Appl Physiol 97: 612-618. [Crossref]

21. Suzuki Y, Ohno S, Okuyama R, Aruga A, Yamamoto M, et al. (2012) Determination of chronic inflammatory states in cancer patients using assay of reactive oxygen species production by neutrophils. Anticancer Res 32: 565-570.

22. Suzuki K, Tomari M, Takahashi M, Sugama K, Otsuka Y, et al. (2012) Assessment of antioxidant and anti-inflammatory actions of curcumin by application of novel neutrophil activity measurement system. Jpn J Clin Chem 41: 343-348.

23. Sugama K, Suzuki K, Yoshitani K, Shiraishi K, Miura S, et al. (2015) Changes of thioredoxin, oxidative stress markers, inflammation and muscle/renal damage following intensive endurance exercise. Exerc Immunol Rev 21: 130-142.

24. Sugama K, Suzuki K, Yoshitani K, Shiraishi K, Kometani T, et al. (2012) IL-17, neutrophil activation and muscle damage following endurance exercise. Exerc Immunol Rev 18: 116-127.

25. Kanda K, Sugama K, Hayashida H, Sakuma J, Kawakami Y, et al. (2013) Eccentric exercise-induced delayed-onset muscle soreness and changes in markers of muscle damage and inflammation. Exerc Immunol Rev 19: 74-87.

26. Suzuki K, Komaba Y, Tomari M, Suzuki Y, Sugama K, et al. (2012) Functional assessment of plant extracts by application of novel neutrophil activity measurement system. Jpn J Complement Altern Med 9: 89-95.

27. Suzuki K, Ohno S, Suzuki Y, Ohno Y, Okuyama R, et al. (2012) Effect of green tea extract on reactive oxygen species produced by neutrophils from cancer patients Anticancer Res 32: 2369-2375.

28. Aggarwal BB, Sung B (2009) Pharmacological basis for the role of curcumin in chronic diseases: an age-old spice with modern targets. Trends Pharmacol Sci 30: 8594. [Crossref]

29. Takahashi M, Suzuki K, Kim HK, Otsuka Y, Imaizumi A, et al. (2014) Effects of curcumin supplementation on exercise-induced oxidative stress in humans. Int J Sports Med 35: 469-475. [Crossref]

30. Kawanishi N, Kato K, Takahashi M, Mizokami T, Otsuka Y, et al. (2013) Curcumin attenuates oxidative stress following downhill running-induced muscle damage. Biochem Biophys Res Commun 441: 573-578. [Crossref]

31. Suzuki K, Yamada M, Kurakake S, Okamura N, Yamaya K, et al. Circulating cytokines and hormones with immunosuppressive but neutrophil-priming potentials rise after endurance exercise in humans. Eur J Appl Physiol 81: 281-287. [Crossref]

32. Peake J, Suzuki K (2004) Neutrophil activation, antioxidant supplements and exerciseinduced oxidative stress. Exerc Immunol Rev 10: 129-141.

33. Peake JM, Suzuki K, Coombes JS (2007) The influence of antioxidant supplementation on markers of inflammation and the relationship to oxidative stress after exercise. $J$ Nutr Biochem 18: 357-371. [Crossref]
34. Kawanishi N, Mizokami T, Niihara H, Yada K, Suzuki K (2016) Neutrophil depletion attenuates muscle injury after exhaustive exercise. Med Sci Sports Exerc 48: 19171924. [Crossref]

35. Kawanishi N, Mizokami T, Niihara H, Yada K, Suzuki K (2016) Macrophage depletion by clodronate liposome attenuates muscle injury and inflammation following exhaustive exercise. Biochem Biophys Rep 5: 146-151. [Crossref]

36. Peake J, Nosaka K, Suzuki K. (2005) Characterization of inflammatory responses to eccentric exercise in humans. Exerc Immunol Rev 11:64-85.

37. Suzuki K, Hashimoto H, Oh T, Ishijima T, Mitsuda H, et al. (2013) The effects of sports drink osmolality on fluid intake and immunoendocrine responses to cycling in hot conditions. J Nutr Sci Vitaminol 59: 206-212.

38. Suzuki K, Shiraishi K, Yoshitani K, Sugama K, Kometani T, et al. (2014) The effect of a sports drink based on highly branched cyclic dextrin on cytokine responses to exhaustive endurance exercise. J Sports Med Phys Fitness 54: 622-630.

39. Ross ML, Halson SL, Suzuki K, Garnham A, Hawley JA (2010) Cytokine response to carbohydrate ingestion during recovery from exercise-induced muscle injury. $J$ Interferon Cytokine Res 30: 329-337.

40. Tanisawa K, Suzuki K, Ma S, Kondo S, Okugawa S, et al. (2018) Effects of ingestion of different amounts of carbohydrate after endurance exercise on circulating cytokines and markers of neutrophil activation. Antioxidants 7: 51. [Crossref]

41. Peake JM, Roberts LA, Figueiredo VC, Egner I, Krog S, et al. (2017) The effects of cold water immersion and active recovery on inflammation and cell stress responses in human skeletal muscle after resistance exercise. J Physiol 595: 695-711. [Crossref]

42. Kawamura T, Suzuki K, Takahashi M, Tomari M, Hara R, et al. (2018) Involvement of neutrophil dynamics and function in exercise-induced muscle damage and delayedonset muscle soreness: Effect of hydrogen bath. Antioxidants 7: 127. [Crossref]

43. Suzuki K, Nakaji S, Yamada M, Totsuka M, Sato K, et al. (2002) Systemic inflammatory response to exhaustive exercise: Cytokine kinetics. Exerc Immunol Rev 8: 6-48.

44. Lim CL, Suzuki K (2017) Systemic inflammation mediates the effects of endotoxemia in the mechanisms of heat stroke. Biol Med (Aligarh) 9: 1000376.

45. Suzuki K, Takahashi M, Li CY, Lin SP, Tomari M, et al. (2015) The acute effects of green tea and carbohydrate co-ingestion on systemic inflammation and oxidative stress during sprint cycling. Appl Physiol Nutr Metabol 40: 997-1003. [Crossref]

46. Li CY, Suzuki K, Hung YL, Yang MS, Yu CP, et al. (2017) Aloe metabolites prevent LPS-induced sepsis and inflammatory response by inhibiting mitogen-activated protein kinase activation. Am J Chin Med 45: 847-861. [Crossref]

47. Hung YL, Fang SH, Wang SC, Cheng WC, Liu PL, et al. (2017) Corylin protects LPSinduced sepsis and attenuates LPS-induced inflammatory response. Sci Rep 7: 46299 [Crossref]

48. Yada K, Suzuki K, Oginome N, Ma S, Fukuda Y, et al. (2018) Single dose administration of taheebo polyphenol enhances endurance capacity in mice. Sci Rep 8: 14625.

49. Ma S (2018) An 8-week ketogenic diet alternated interleukin-6, ketolytic and lipolytic gene expression, and enhanced exercise capacity in mice. Nutrients 10: 1696.

50. Suzuki K (2017) Exhaustive exercise-induced neutrophil-associated tissue damage and possibility of its prevention. J Nanomedicine Biotherapeutic Discov 7: 156.

51. Suzuki K (2018) Cytokine response to exercise and its modulation. Antioxidants 7: 17

52. Hung YL, Suzuki K (2017) The pattern recognition receptors and lipopolysaccharides (LPS)-induced systemic inflammation. Int J Res Studies Med Health Sci 2: 1-7.

53. Ma S, Suzuki K (2018) Toll-like receptor 4: Target of lipotoxicity and exerciseinduced anti-inflammatory effect? Ann Nutr Food Sci 2: 1027.

Copyright: (C2018 Suzuki K. This is an open-access article distributed under the terms of the Creative Commons Attribution License, which permits unrestricted use, distribution, and reproduction in any medium, provided the original author and source are credited. 